Non-linear Fractional Polynomials for Estimating Long-Term Persistence of Induced Anti-HPV Antibodies: A Hierarchical Bayesian Approach Peer-reviewed author version

AREGAY, Mehreteab; SHKEDY, Ziv; MOLENBERGHS, Geert; David, Marie-Pierre \& TIBALDI, Fabian (2014) Non-linear Fractional Polynomials for Estimating Long-Term Persistence of Induced Anti-HPV Antibodies: A Hierarchical Bayesian Approach. In: STATISTICS IN BIOPHARMACEUTICAL RESEARCH, 6 (3), p. 199-212.

DOI: $10.1080 / 19466315.2014 .911201$

Handle: http://hdl.handle.net/1942/17695 


\title{
Non-linear Fractional Polynomials for Estimating Long-Term Persistence of Induced anti-HPV Antibodies: A Hierarchical Bayesian Approach.
}

\author{
Mehreteab Aregay $^{1}$ Ziv Shkedy ${ }^{2}$ Geert Molenberghs ${ }^{1,2}$ \\ Marie-Pierre David ${ }^{3}$ Fabian Tibaldi ${ }^{3}$ \\ ${ }^{1}$ I-BioStat, Katholieke Universiteit Leuven, B-3000 Leuven, Belgium \\ ${ }^{2}$ I-BioStat, Universiteit Hasselt, B-3590 Diepenbeek, Belgium \\ ${ }^{3}$ GlaxoSmithKline Biologicals, 89 Rue de l'Institut, B-1330, Rixensart, Belgium
}

\begin{abstract}
When the true relationship between a covariate and an outcome is non-linear, one should use a nonlinear mean structure that can take this pattern into account. In this paper, the fractional polynomial modeling framework, which assumes a pre-specified set of powers, is extended to a non-linear fractional polynomial framework (NLFP). Inferences are drawn in a Bayesian fashion. The proposed modeling paradigm is applied to predict the long-term persistence of vaccine induced anti-HPV antibodies. In addition, the subject-specific posterior probability to be above a threshold value at a given time is calculated. The model is compared with a power-law model using the Deviance Information Criterion (DIC). The newly proposed model is found to fit better than the power-law model. A sensitivity analysis was conducted, from which a relative independence of the results from the prior distribution of the power was observed.
\end{abstract}

Some Keywords: Deviance information criterion; Fractional polynomial model; Non-linear fractional polynomial; Power-law model

\section{Introduction}

Human papillomavirus (HPV) infection is a necessary cause of cervical cancer. Even though $90 \%$ of the HPV infections are cleared within two years (Goldstein et al., 2009), persistent infection will lead to the development of cervical cancer and other anogenital cancers (Ho et al., 1998). There are 120 HPV types which are identified and indexed by a number (Chaturvedi and Maura, 2010). Of more than 40 HPV types, HPV-16 and HPV-18 cause about 70\% of the cervical cancers (Muñoz et al., 2003).

To protect against persistent HPV infection, many scientists synthesized a virus-like particle (VLP) vaccine (Zhou et al., 1991; Kirnbauer et al., 1992). There are two types of vaccines available on the market, Cervarix 
and Gardasil $^{1}$, that prevent infection with HPV-16/18 and may lead to further decrease in cervical cancer (Kahn, 2009). Several authors employed different models to predict long-term immunity following this vaccine and/or natural infection. Fraser et al. (2007) proposed a modified power-law model, which imposes an asymptote onto the antibody level, whereas David et al. (2009) implemented a piece-wise model, which assumes a constant antibody decline within a specified period. Recently, Aregay et al. (2013) showed that the power-law model of Fraser et al. (2007) and David et al. (2009) can be formulated using fractional polynomial (FP), which is a data-driven method to predict long-term persistence and to estimate the time point above a given threshold.

Royston and Altman (1994) extended conventional polynomial regression to the aforementioned FPs, a more flexible method. It has been shown that FPs are frequently among the least biased smoothing methods for fitting non-linear exposure effects (Govindarajulu et al., 2009). Several researchers applied FPs to non-linear longitudinal data (Long and Ryoo, 2010). Unsurprisingly, there are also some limitations to FP functions. Some of them are sufficiently flexible to capture a non-linear function and possible sensitivity to extreme values at either end of the distribution of a covariate (Royston and Sauerbrei, 2008). Although Royston and Sauerbrei (2008) argued that the set $\{-2,-1,-0.5,0,0.5,1,2,3\}$ is oftentimes sufficient to approximate all powers in the interval $[-2,3]$, there may be reasons to extend it (Shkedy et al., 2006; Aregay et al., 2013).

To incorporate prior information, Bové and Held (2010) implemented an FP model that combines variable selection and "parsimonious parametric modeling" (Royston and Altman, 1994) of the covariate effects, with Bayesian methods for univariate data. Auranenn et al. (1999) fitted a hierarchical Bayesian regression model to predict the duration of immunity to Hemophilus influenza type b. In the context of long-term antibody persistence after vaccination of Hepatitis A, Van Damme et al. (1994) used the geometric mean antibody titer (GMT) to show that the predicted duration of antibody persistence for inactivated hepatitis A vaccine is estimated to be at least 20 years. Wiens et al. (1996) proposed to estimate duration of protection for the Hepatitis A vaccine based on an individual level instead of the GMT level. In the current paper, we combined the approaches of Van Damme et al. (1994) and Wiens et al. (1996) and use a hierarchical Bayesian model from which we estimate both the mean antibody titer and subject-specific profiles. Long-term persistence is

\footnotetext{
${ }^{1}$ Cervarix is a registered trade mark of the GlaxoSmithKline group of companies and Gardasil is a registered trade mark of Merck and Co Inc.
} 
estimated using information obtained from subject-specific profiles.

In this paper, we extend the FP framework to non-linear longitudinal data using a hierarchical Bayesian approach. The method is applied to predict the long-term persistence of vaccine-induced anti-HPV-16 and anti-HPV-18 antibodies, as well as to predict the proportion of subjects above a threshold value. Many researchers (Fraser et al., 2007; David et al., 2009; Aregay et al., 2013) have been focusing on the prediction of long-term immunity but no attention was given to the subject-specific probability of being above a threshold for a given time point. In contrast with the model-based long-term prediction, which treats subjects as above threshold or not at any given time point, a subject-specific probability quantifies the uncertainty about the subject protection status at any time point.

The paper is organized as follows. Section 2 introduces the data set and a brief exploratory data analysis. The hierarchical Bayesian model used to predict the probability of being above a threshold is discussed in Section 3. We apply the proposed model to the data in Section 4 and discuss the results in Section 5.

\section{Motivating Case Study}

The data set used encompasses 390 healthy women who received the HPV-16/18 AS04-adjuvanted vaccine (Cervarix). They were enrolled in the initial multi-center study (HPV-001, NCT00689741) and took part in a follow-up study for three additional years (HPV-007, NCT00120848). Their blood samples were evaluated for the presence of anti-HPV-16/-18 antibodies at months 7, 12, 18 and then annually up to 6.4 years. The age of the women ranges over 15-25 years and they came from North America (USA and Canada) and Brazil. An enzyme linked immunosorbent assay (ELISA) which was developed in-house by GlaxoSmithKline (GSK) was used to check the presence of anti-HPV-16/-18 antibodies. The assay cut-off value was $8 \mathrm{EU} / \mathrm{mL}$ and 7 $\mathrm{EU} / \mathrm{mL}$ for anti-HPV-16 and anti-HPV-18 antibodies, respectively.

The number of blood samples for each visit (categorized month; the continuous time in month was categorized into 12 time points) was obtained. For anti-HPV-16 antibodies, there was a drop in the number of blood samples in the month range 25-32. Month 7 reveals the highest number of blood samples and thereafter a

small increase from 364 to around 366 was shown in month 12 and 18, but there was a rapid decrease to 

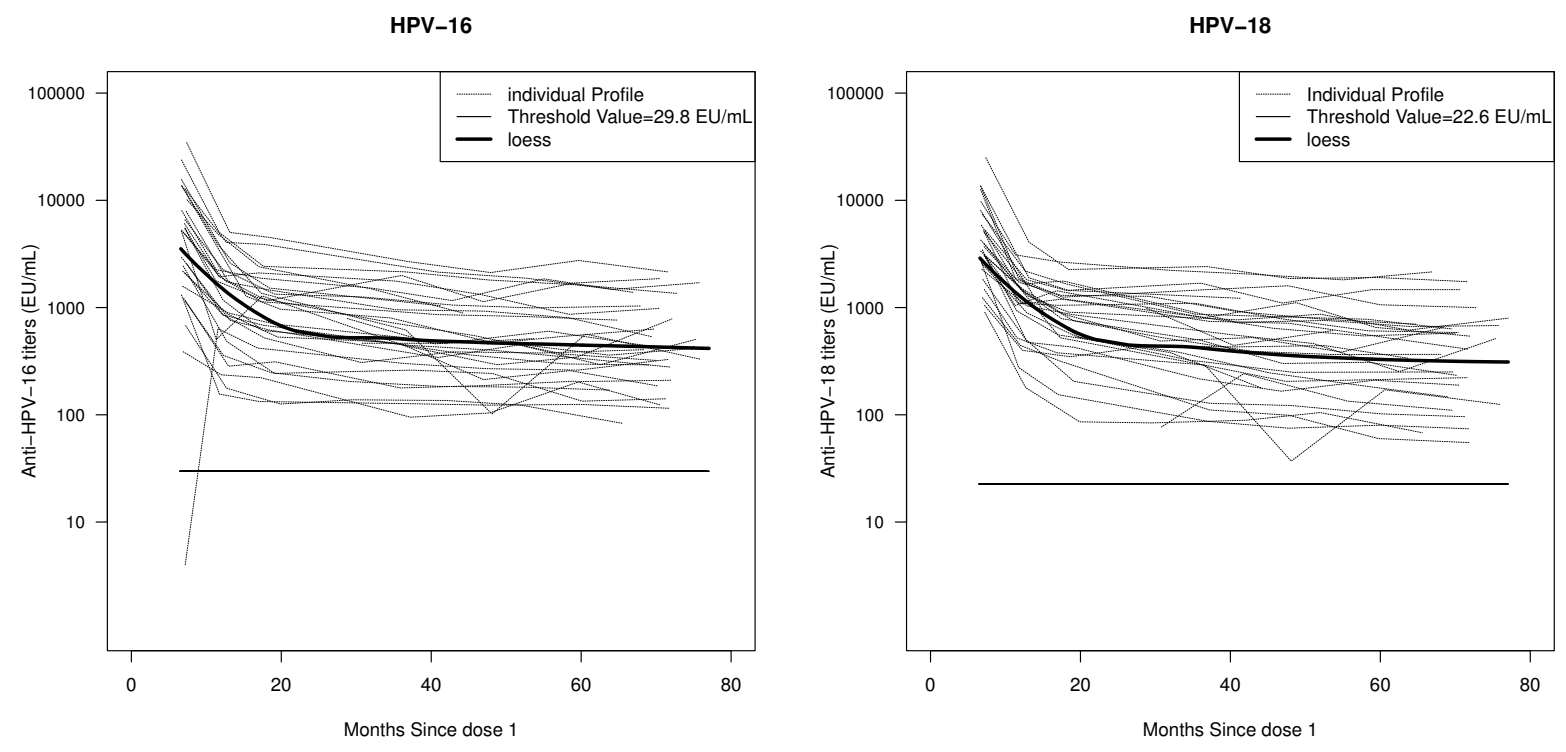

Figure 1: Random selection of 30 individual profiles for HPV-16 (left panel) and for HPV-18 (right panel).

89 in the months 25-32. The number of blood samples ranges between 130 and 234 in the month range 69-74, followed by a decrease to 66 blood samples in months beyond 75. A similar pattern was observed for anti-HPV-18 antibodies.

Figure 1 shows randomly selected individual profile curves for the antibody mean and threshold value for both HPV-16 and HPV-18 and reveals a high amount of variability between subjects, as well as that there are a few subjects who cross a threshold value. The evolutions of mean antibody over time for both HPV-16 and HPV-18 are shown in Figure 2. In both studies, it can be seen that the decline in the antibody level was higher in the first few months followed by a moderate decrease until the end of the follow-up period.

\section{Modeling Mean Antibody Using Hierarchical Subject-specific Models}

\subsection{Model Formulation}

In previous studies of the decline in antibody level after induced vaccination against HPV, Fraser et al. (2007) and David et al. (2009) employed a power-law (PL) model to estimate the persistence of anti-HPV level. 

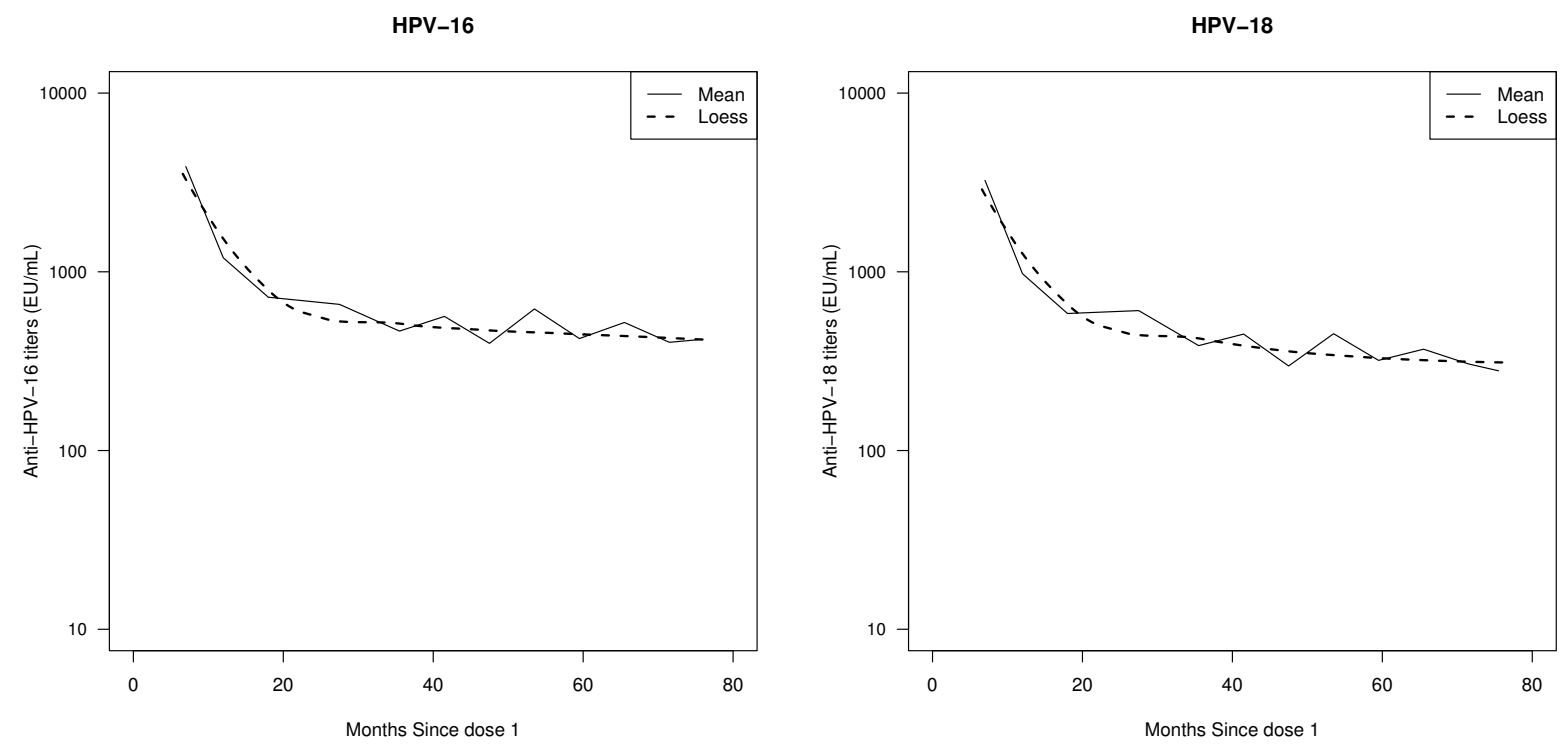

Figure 2: Mean structure of HPV-16 and HPV-18.

We start this section with a brief discussion the modelling approaches for longitudinal anti-HPV antibodies data and then we formulate the hierarchical Bayesian model used for the analysis presented in this paper. Let $Y_{i j}$ denote the $\log$ antibody level of subject $i$ measured at time $j$, and consider the model

$$
Y_{i j}=f\left(t_{i j}\right)+\varepsilon_{i j}
$$

$\left(i=1, \ldots, n ; j=1, \ldots, t_{i}\right)$. Here, $f\left(t_{i j}\right)$ is the mean log antibody titer, assumed to be time-dependent, and $\varepsilon_{i j}$ is the random error term for subject $i$ at time $j$, assumed to be normally distributed, $\varepsilon_{i j} \sim N\left(0, \sigma_{\varepsilon}^{2}\right)$. The mean structure of such a subject-specific power-law model, discussed by Fraser et al. (2007), David et al. (2009), and Aregay et al. (2013) could be formulated as

$$
f\left(t_{i j}\right)=\left(\beta_{0}+b_{0 i}\right)+\left(\beta_{1}+b_{1 i}\right) \ln t_{i j}
$$

Note that the parameters $\beta_{0}$ and $\beta_{1}$ are the population (fixed) parameters, while $b_{0 i}$ and $b_{1 i}$ are subjectspecific random intercept and random slope, respectively, $b_{0 i} \sim N\left(0, \sigma_{b_{0}}^{2}\right)$ and $b_{1 i} \sim N\left(0, \sigma_{b_{1}}^{2}\right)$.

Aregay et al. (2013), pointed out that the power-law model can be formulated as first-order fractional polynomials with $p=0$ using the Box-Tidwell transformation (Box and Tidwell, 1962). For the general case with $p \neq 0$ Aregay et al. (2013) used a first-order fractional polynomial mixed model to model the long-term 
persistence of induced anti-HPV antibodies in which the mean structure is given by:

$$
f\left(t_{i j}\right)=\left(\beta_{0}+b_{0 i}\right)+\left(\beta_{1}+b_{1 i}\right) t_{i j}^{p}
$$

Within the fractional polynomials framework, the unknown power in (3) are estimated by a grid search over the pre-specified sequence $p_{1} \leq p_{2} \leq \cdots \leq p_{m}$. Note that, for a given value of $p$, the mean structure in (3) is linear with respect to $t_{i j}^{p}$. Aregay et al. (2013) compared the first- and second-order fractional polynomials for the data set discussed in Section 2 and they found that the first order fractional polynomial in (3) was the best model. In what follows, we formulate a hierarchical Bayesian model that, in contrast with the FP framework, is estimating the FP model (3) as a non-linear model (NLFP).

At the first stage of the hierarchical model we specify a normal likelihood model, i.e.,

$$
Y_{i j} \sim \mathrm{N}\left(f\left(t_{i j}\right), \sigma_{\varepsilon}^{2}\right)
$$

where $f\left(t_{i j}\right)$ has the mean structure defined in (3). In the second stage of the model we specify a prior model for the unknown parameters in the model. We considered independent normal prior distributions for the population parameters,

$$
\boldsymbol{\beta}_{k} \sim N\left(\mu_{k}, \sigma_{\beta_{k}}^{2}\right), \quad \text { for } k=0,1 .
$$

The subject-specific intercept and slope are assumed to follow a bivariate normal distribution with a mean vector of zeros and variance-covariance structure $\mathbf{D}_{b}$, i.e.,

$$
\left[\begin{array}{c}
b_{0 i} \\
b_{1 i}
\end{array}\right] \sim \operatorname{MVN}\left(\left[\begin{array}{l}
0 \\
0
\end{array}\right], \mathbf{D}_{b}=\left[\begin{array}{ll}
\sigma_{b_{0}}^{2} & \rho_{b_{0} b_{1}} \sigma_{b_{0}} \sigma_{b_{1}} \\
\rho_{b_{0} b_{1}} \sigma_{b_{0}} \sigma_{b_{1}} & \sigma_{b_{1}}^{2}
\end{array}\right]\right) .
$$

A uniform prior distribution was defined for the power $p \sim \mathrm{U}(a, b)$. A sensitivity analysis for the choice of $a$ and $b$ is performed and is presented in Section 4.1.

In order to complete the specification of the hierarchical model, the following hyperprior distributions were specified. A non-informative independent normal prior distribution (Gelman, 2006) for $\mu_{k}$ and a gamma prior distribution (Gelman, 2006; Spiegelhalter et al., 2003) for the precision parameters, i.e., $\sigma_{\beta_{k}}^{-2} \sim G(0.01,0.01)$, and $\sigma_{\varepsilon}^{-2} \sim G(0.01,0.01)$. However, Gelman (2006) argued that this prior is informative because of its shape and suggested to use instead a uniform prior on the hierarchical standard deviation. We have considered a uniform prior distribution for the standard deviation but the result does not change much compared with 
the result obtained from the assumption of an inverse gamma prior for the variance. An inverse Wishart prior distribution for the covariance matrix $\mathbf{D}_{b}$ was employed, i.e.,

$$
\mathbf{D}_{b}^{-1} \sim \text { Wishart }\left(R_{D}, 2\right)
$$

where $R_{D}$ is $2 \times 2$ identity matrix. We notice that the case in which $\mathbf{D}_{b}$ is diagonal, i.e., the random intercept an slope are independent, implies that

$$
b_{0 i} \sim N\left(0, \sigma_{b_{0}}^{2}\right) \text { and } b_{1 i} \sim N\left(0, \sigma_{b_{1}}^{2}\right)
$$

with hyperprior distribution given by $\sigma_{b_{k}}^{-2} \sim G(0.01,0.01)$ for $k=0,1$.

Model formulation for the modified power-law model is given in Section 3 in the Supplementary Web Appendix of this paper

\subsection{The Probability above a Threshold}

All studies discussed above were conducted to assess the long-term protection after vaccination. Such an assessment can be done by comparing the model-based empirical Bayes subject-specific predictions at a given time point to a pre-specified threshold $\tau$. Based on this approach, Fraser et al. (2007), David et al. (2009), and Aregay et al. (2013) concluded that $76 \%, 100 \%$, and $99.7 \%$ of the vaccinated subjects will be above the threshold level near life long persistence of anti-HPV-16 antibodies after vaccination.

One of the motivations for using the hierarchical Bayes model presented in the previous section is to calculate the probability of being above a threshold for a given time point. Note that, in contrast with the approaches discussed by Fraser et al. (2007), David et al. (2009), and Aregay et al. (2013), here we wish to quantify the uncertainty about being above threshold or not, rather than just the status of each subject. Hence, for each subject, we estimate the probability of being above a pre-specified threshold. Let $Z_{i j}$ be an indicator latent variable, representing above threshold or not status of the $i$ th subject at time $t_{i j}$ :

$$
Z_{i j}= \begin{cases}1 & Y_{i j}>\tau, \text { an antibody level above a threshold } \\ 0 & Y_{i j} \leq \tau, \text { an antibody level below a threshold }\end{cases}
$$

Hence, the subject-specific probability of being above a given threshold value at time $t_{i j}$ is given by $\pi_{i j}=$ $P\left(Y_{i j}>\tau\right)$. We notice that to estimate the proportion of subjects above a threshold in the sample, Fraser 
et al. (2007), David et al. (2009), and Aregay et al. (2013) derive the value of $Z_{i j}$ as

$$
\widehat{Z}_{i j}= \begin{cases}1 & \widehat{f}\left(t_{i j}\right)>\tau, \\ 0 & \widehat{f}\left(t_{i j}\right) \leq \tau\end{cases}
$$

Hence, the proportion of subjects above a threshold at time $t_{i j}$ was estimated by

$$
\bar{p}_{j}=\sum_{i=1}^{n_{j}} \widehat{Z}_{i j} / n_{j},
$$

where $n_{j}$ is the number of subjects at the $j$ th time point. The proposed hierarchical Bayes model allows us to estimate the probability $\pi_{i j}$ using Monte Carlo integration. In practice, $\pi_{i j}$ can be obtained from the WinBUGS program by using the step( ) function. The function creates a binary variable that counts the number of MCMC simulations for which, $Y_{i j}>\tau$ is true. Hence, the $0 / 1$ values from the step( ) function can be used to compute $\pi_{i j}$. For example, consider an MCMC simulation with $M$ samples of step $\left(Y_{i j}\right)$ from which the first $T$ are the burn-in samples, $\pi_{i j}$ is estimated using Monte Carlo integration by

$$
\frac{1}{M} \sum_{t=T+1}^{T+M} Z_{i j}^{(t)} .
$$

This implies that the hierarchical Bayesian model enables estimation of both the quantities $Z_{i j}$ and $\pi_{i j}$.

\section{Data Application}

\subsection{Long-term Prediction Using Subject-specific Non-linear Fractional Polyno- mials}

To estimate the subject evolution of the log antibodies within the follow up period, the NLFP model was fitted using the R2WinBUGS package (Sturtz et al., 2005). A Markov Chain Monte Carlo (MCMC) simulation of 10,000 iterations, from which the first 1000 were considered burn in and discarded from the analysis, was used to estimate the model parameters. Model selection was done using the Deviance Information Criterion (DIC; Spiegelhalter et al. , 2002) and convergence was checked using trace plots, estimated potential scale reduction factor $(\widehat{R})$ and Brooks, Gelman, and Rubin's (BGR) statistics (Gelman and Rubin, 1992). The trace plot and BGR statistic indicate convergence for all model parameters. Furthermore, the estimated

potential scale reduction factor $(\widehat{R})$ for all the parameters were close to one which indicates convergence for all model parameters (see Supplementary Web Appendix). 
Initially, we fitted an NLFP that assumes the random intercept and the random slope to be independent. Because we have prior knowledge of the power $p$, which is $p=-1.25$ from Aregay et al. (2013), a uniform prior distribution for the power $p \sim \mathrm{U}(-1.4,-1.2)$ was used. A sensitivity analysis for the prior of $p$ will be discussed in the section below. The posterior mean for the power was estimated to be -1.356 for anti-HPV-16 antibodies, while -1.259 for anti-HPV-18 antibodies. In the second stage, an NLFP that assumes the random intercept and the random slope to be correlated was applied. For this model, the posterior means are estimated to be equal to -1.332 for anti-HPV-16 antibodies, whereas it is -1.243 for anti-HPV-18 antibodies. For both anti-HPV-16 and anti-HPV-18 antibodies, the DIC for the correlated random-effects model was smaller than the DIC for the independent random effects model. Hence, the former is to be preferred. Additionally, the posterior mean for the correlation between the random effects is equal to $-0.413(95 \%$ credible interval: $[-0.511,-0.307])$ for anti-HPV-16 antibodies and -0.596 (credible interval $[-0.677,-0.508])$ for anti-HPV-18 antibodies. Parameter estimates for the posterior mean of the fixed effects are shown in Table 1.

To assess whether the results depend on the prior distribution of the power $p$, a sensitivity analysis was performed using different values for $a$ and $b$. This shows that the results do not depend on the prior distribution chosen (see Table 2).

Different threshold values $(\tau)$ were used: $29.8 \mathrm{EU} / \mathrm{mL}(1.474 \log (\mathrm{EU} / \mathrm{mL}))$ and $417.8 \mathrm{EU} / \mathrm{mL}(2.621 \log$ $(\mathrm{EU} / \mathrm{mL}))$ for anti-HPV-16 antibodies and $22.6 \mathrm{EU} / \mathrm{mL}(1.355 \log (\mathrm{EU} / \mathrm{mL}))$ and $279.3 \mathrm{EU} / \mathrm{mL}(2.446 \log$ (EU/mL)) for anti-HPV-18 antibodies (Fraser et al., 2007; David et al., 2009; Aregay et al., 2013). Unless otherwise specified in the text, authors will focus their analysis on the low threshold values, i.e., $29.8 \mathrm{EU} / \mathrm{mL}$ for anti-HPV-16 antibodies and 22.6 EU/mL for anti-HPV-18 antibodies.

Figure 3 shows the long-term posterior predicted population means with $95 \%$ predictive intervals, indicating that on average all of the subjects have antibody levels above a threshold level for near life time.

Figure 4 shows the observed and posterior prediction for the antibody levels for selected months within the estimation period. The posterior prediction densities and observed densities are similar, indicating that the NLFP is fitting the data very well over the follow-up period. The posterior predictive densities for 30 and 

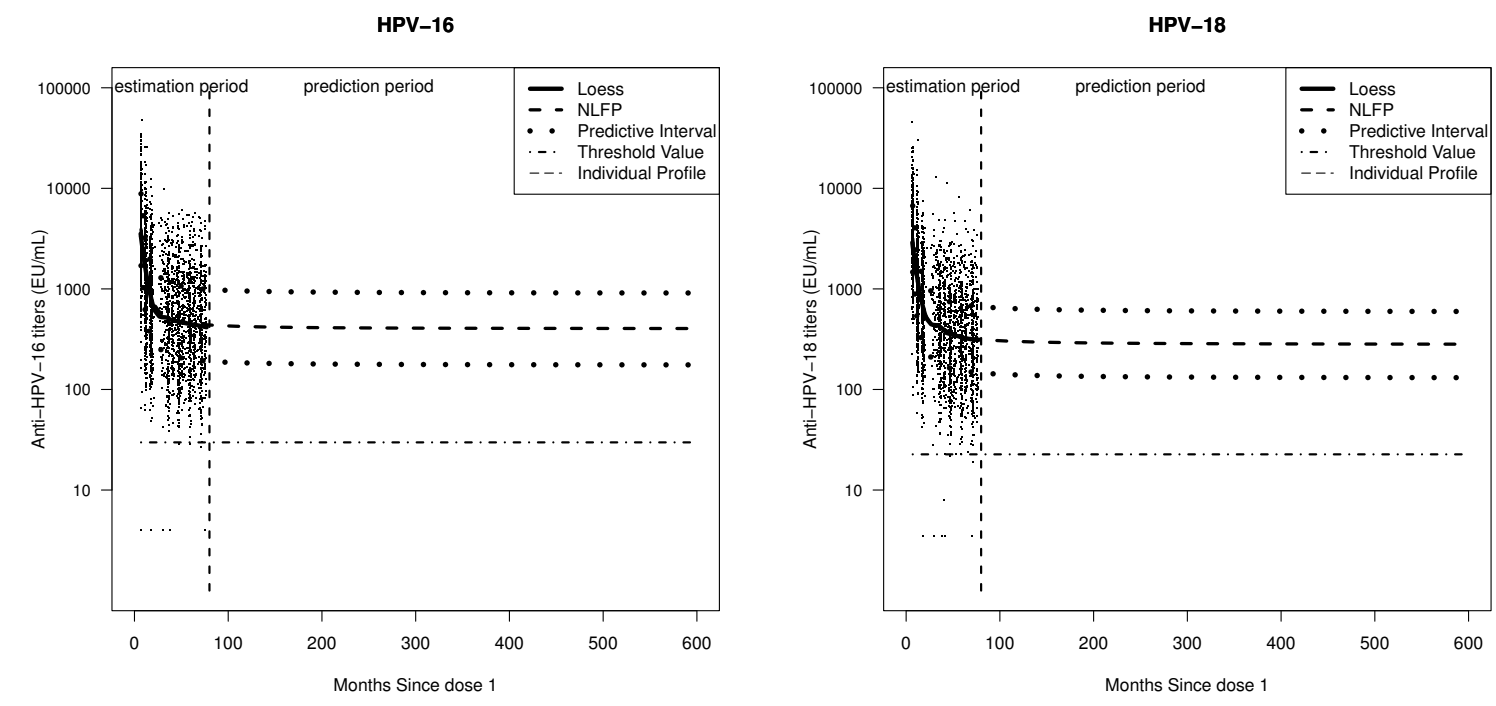

Figure 3: Long-term prediction with posterior predictive interval over 50 years for anti-HPV-16 (left panel) and anti-HPV-18 antibodies (right panel).

50 years at different threshold values are shown in Figure 5. Note that the posterior predictive densities for 30 years and 50 years are almost the same.

A comparison of the observed and model-based proportion within the follow up period using the power-law and NLFP model is shown in Figure 6 at the top. Using the NLFP, we observe from the lower panels that the proportion of subjects who are above the threshold value was $99.7 \%$ (389 out of 390 subjects) for anti-HPV-16 antibodies, while it was $99.5 \%$ (388 out of 390 subjects) for anti-HPV-18 antibodies over 50 years. If we use $\tau=2.621$ and $\tau=2.446$, the proportion above the threshold will decrease to $48.9 \%$ and 52.6\% for anti-HPV-16 and anti-HPV-18 antibodies, respectively. These results agree with these reported in David et al. (2009) and Aregay et al. (2013)

\subsection{Estimation of Subject-specific Probability to be Above a Threshold}

As we mentioned in Section 3.2, the hierarchical Bayesian model allows us to estimate the posterior probability of being above a threshold for a given time point. First, we discuss the results obtained for the anti-HPV-16 antibodies.

The posterior mean for some selected subjects is shown in Figure 7. Note that the first subject (8650) has 


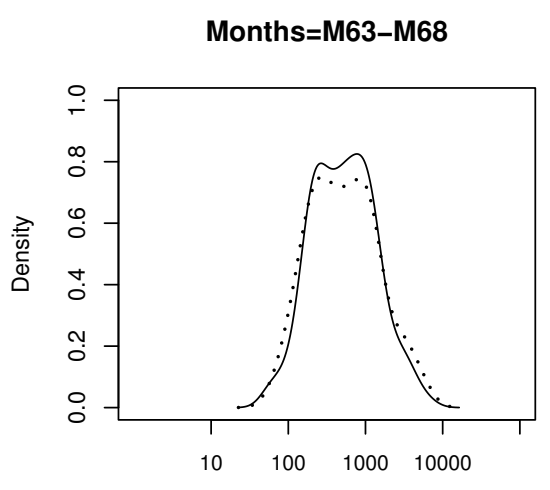

Anti-HPV-16 titers (EU/mL)

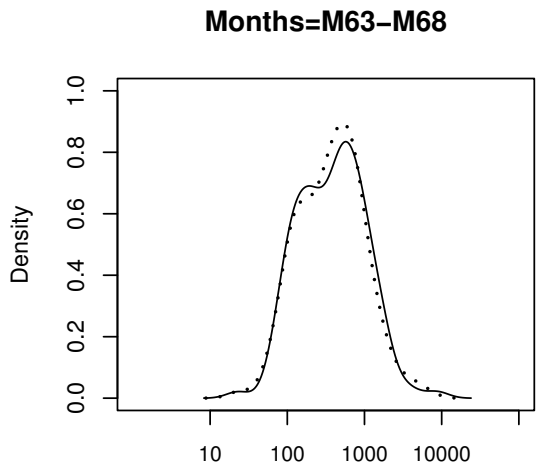

Anti-HPV-18 titers (EU/mL)
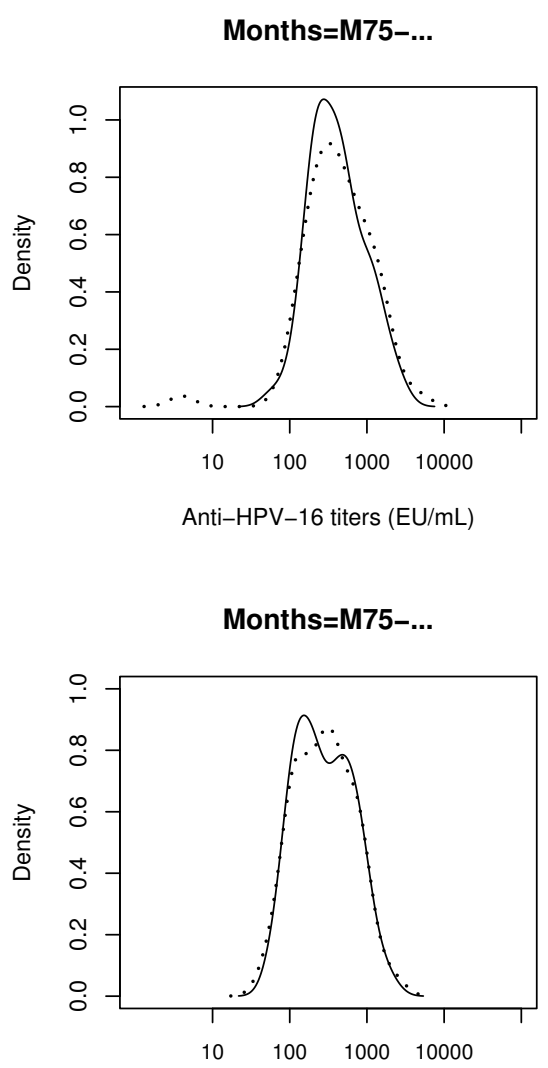

Anti-HPV-18 titers (EU/mL)

Figure 4: The densities of the posterior predictions (solid line) and observed values (dashed line) of the antibody level at the categorized months, i.e., M63-M68 and M75-.., for anti-HPV-16 (top figure) and anti-HPV-18 antibodies (bottom figure).

a posterior predictive value below the threshold level while the other subjects have values above this one. The estimated posterior mean for $\pi_{i j}$ above the threshold level for these subjects is equal to $0.25,1,1,1$, respectively.

Using the methodology described in Fraser et al. (2007), David et al. (2009) and Aregay et al. (2013), subject 8650 is classified as having a predicted mean below the threshold level for 50 years. However, using the current model $\widehat{\pi}_{i 50}$ is equal to 0.25 . In other words, the current model provides a measure for uncertainty for each subject.

Figure 8 shows the histogram of the posterior probability of being above threshold for anti-HPV-16 antibod- 

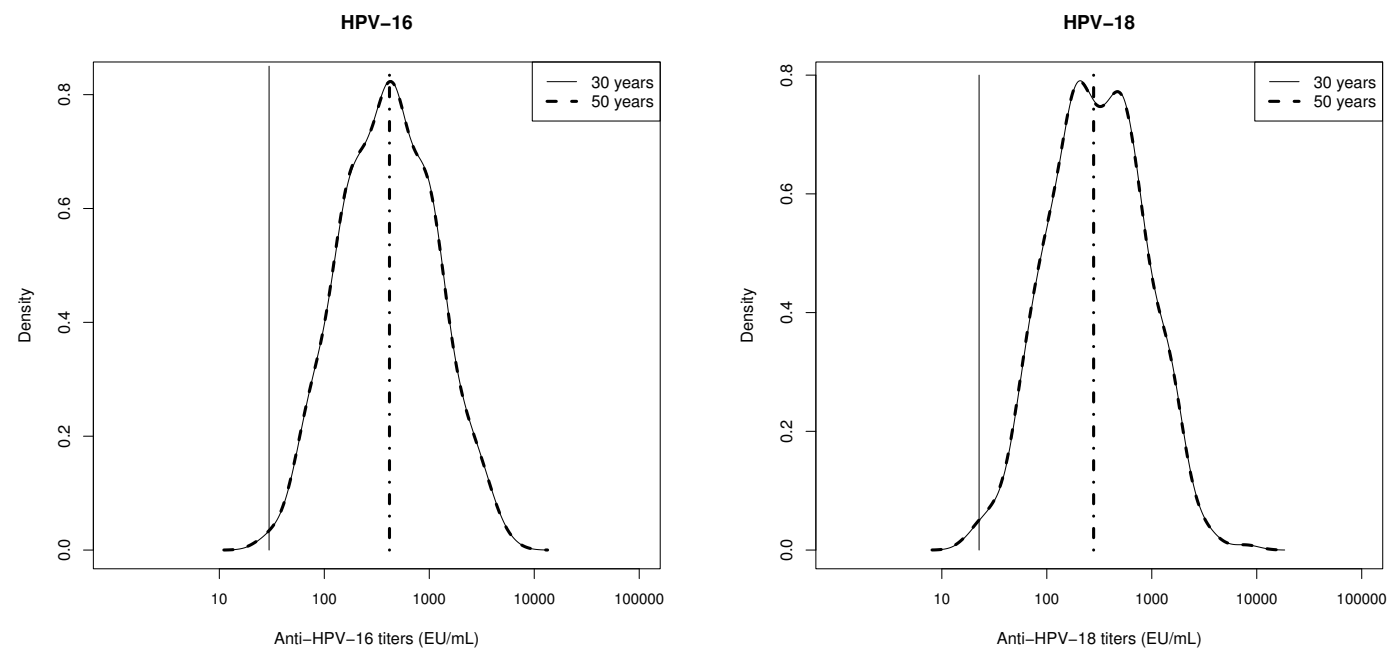

Figure 5: The densities of posterior predictions of the antibody level over 30 and 50 years for anti-HPV-16 and anti-HPV-18 antibodies. Vertical solid line and bold dashed line indicate the thresholds, 29.8 EU/mL and $417.8 \mathrm{EU} / \mathrm{mL}$ (left panel), and $22.6 \mathrm{EU} / \mathrm{mL}$ and $279.3 \mathrm{EU} / \mathrm{mL}$ (right panel), respectively.

ies. It shows that $93(23.8 \%)$ subjects have a posterior probability of being above the threshold $\tau=2.621$ equal to 0 , for 193 (49.5\%), $0 \leq \widehat{\pi}_{i j} \leq 1$ and the rest (104, $26.7 \%$ ) have a probability of being above the threshold equal to $1 ; \widehat{\pi}_{i j}=1$ for 50 years. The sorted posterior probabilities of being above the threshold for all subjects in the trial are shown in left panel of Figure 9 and the posterior probabilities of being above the threshold for 10 years for subjects who have posterior predicted mean above/below the threshold are shown in the right panel of Figure 9 and illustrate the main difference between the analysis presented in this paper to the analysis discussed in Fraser et al. (2007), David et al. (2009), and Aregay et al. (2013). As mentioned before, all authors used a model based classification procedure. In contrast, the right panel of Figure 9 shows $\widehat{\pi}_{i j}$ for subjects who were classified as above/below the threshold by David et al. (2009) and Aregay et al. (2013). We clearly see that among the 199 (51.02\%) subjects who were classified as above the threshold, 102 subjects have $\widehat{\pi}_{i j}=1$, while 97 subjects have $0.5<\widehat{\pi}_{i j}<1$. On the other hand, among the $191(48.98 \%)$ subjects who were classified as below the threshold, 89 subjects have $\widehat{\pi}_{i j}=0$ while 102 subjects have $0<\widehat{\pi}_{i j}<0.5$ over 10 years. This indicates that some of the subjects who were classified as above the threshold in David et al. (2009) and Aregay et al. (2013), are surrounded by some uncertainty.

The hierarchical model allows us to calculate the proportion of individuals for which the probability of being 
HPV-16

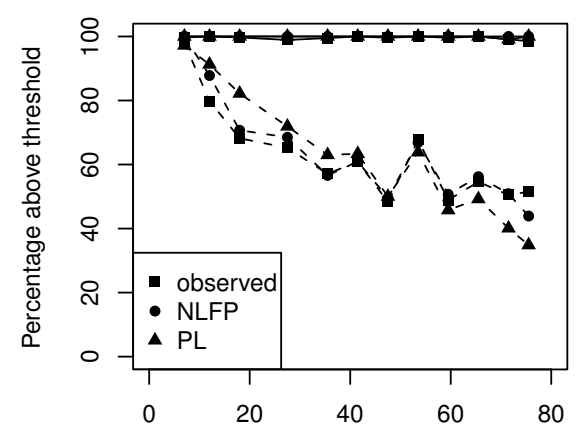

Months Since dose 1

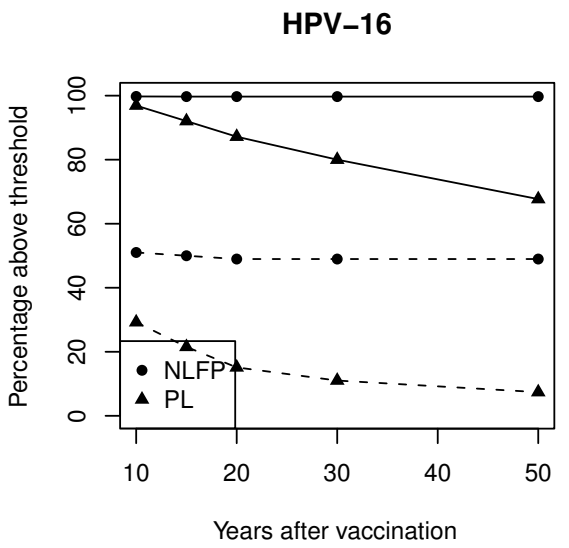

HPV-18

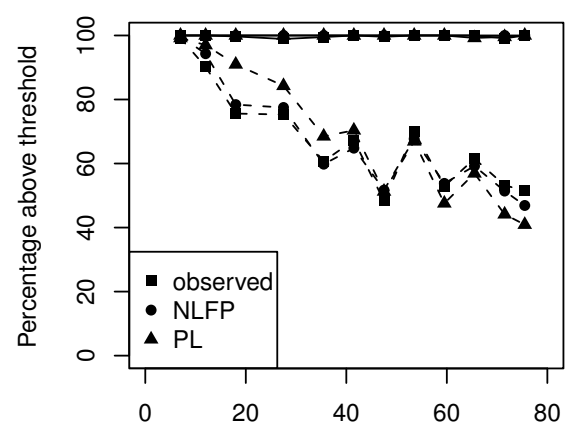

Months Since dose 1

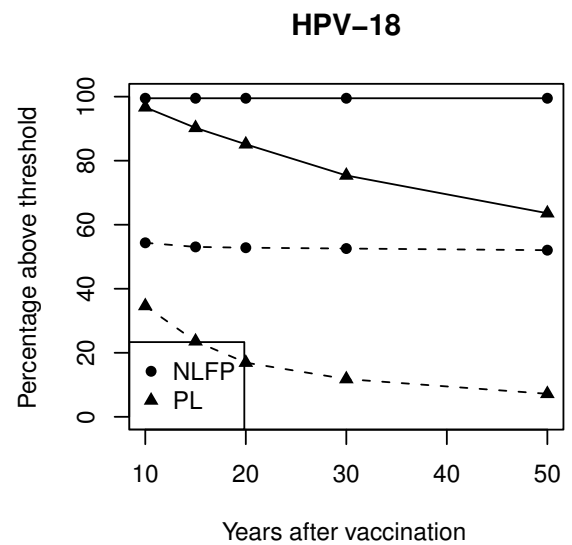

Figure 6: Observed proportion and model-based proportion above different threshold values $(\tau=1.474$ and $\tau=1.355$ (solid line) and $\tau=2.621$ and $\tau=2.446$ (dashed line) for anti-HPV-16 (left panel) and antiHPV-18 antibodies (right panel) within (top figure) and after (bottom figure) the follow up period, using the power-law and NLFP models.

above a threshold is more than $\alpha$. For instance, over 50 years, if we use $\tau=2.621,190$ subjects for antiHPV-16 antibodies have a posterior probability of being above a threshold more than 0.5 . On the other hand, if we use the lower thresholds, $\tau=1.474$, all of the subjects but one have a posterior probability above 0.5 over 50 years.

Figure 10 shows the subject-specific posterior probability of being above a threshold for anti-HPV-18 antibodies. Similar to the results obtained for anti-HPV-16 antibodies, among the $212(54.4 \%)$ subjects who were classified as above the threshold, we can see that 133 subjects have $\widehat{\pi}_{i j}=1$ while 79 subjects have 
Table 1: Comparison of power-law model, non-linear fractional polynomial model with $\rho_{12}=0$ and nonlinear fractional polynomial model with $\rho_{12} \neq 0$ for anti-HPV-16 and anti-HPV-18 antibodies.

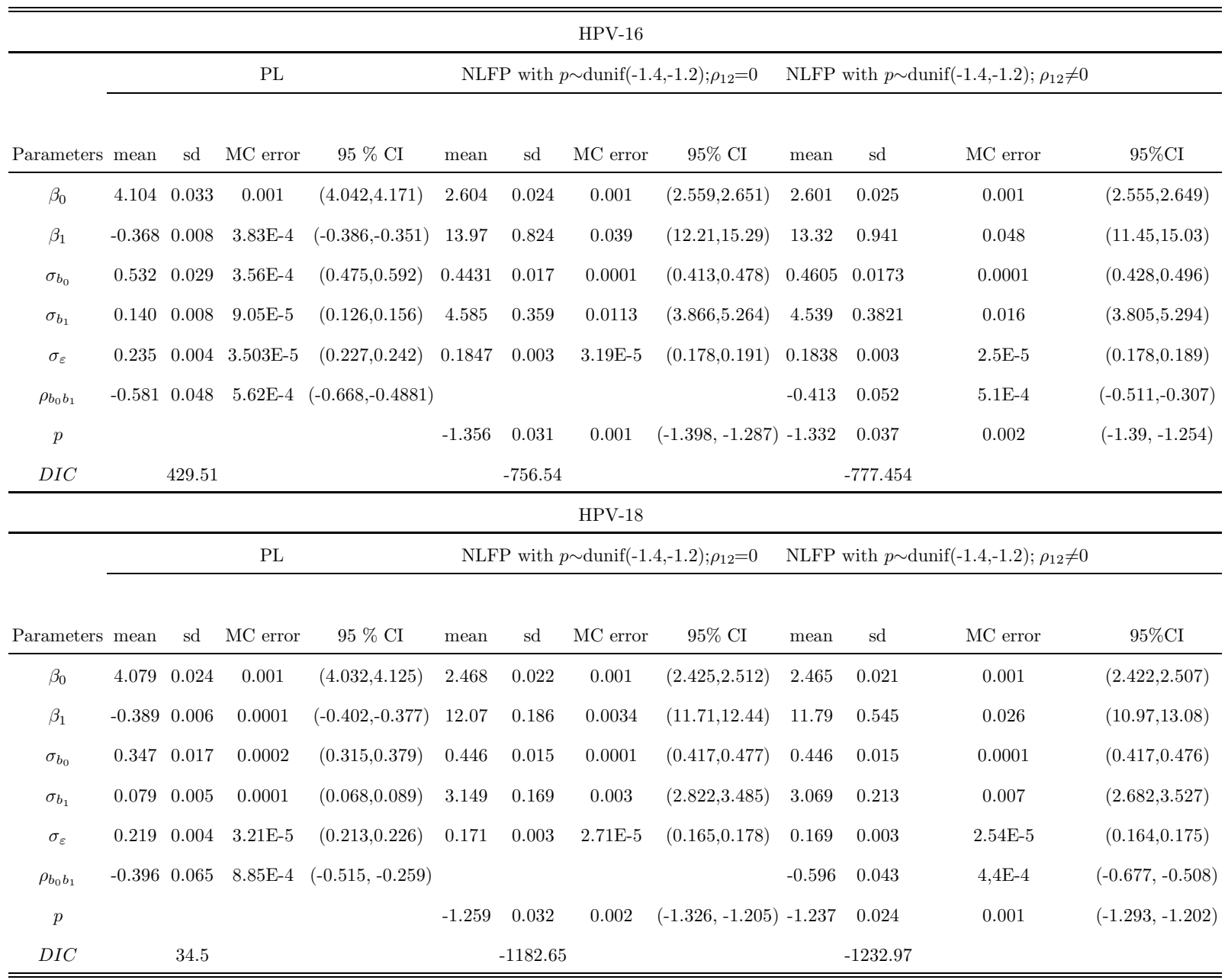

$0.48<\widehat{\pi}_{i j}<1$. For an elaborate presentation of the results obtained for anti-HPV-18 antibodies, we refer to Section 1 in the Supplementary Web Appendix.

There are two vaccines against HPV available on the market. It is difficult for many medical experts to choose among them. Our method can be used to compare two or more vaccines using the posterior probability of being above threshold for a given time point. To underscore this, we created an illustrative figure with vaccine 1 and vaccine 2 (Figure 11). From the plot, we can see that vaccine 1 is better than vaccine 2 . 
Table 2: A sensitivity analysis of the non-linear fractional polynomial for anti-HPV-16 and anti-HPV-18 antibodies.

\begin{tabular}{|c|c|c|c|c|c|c|c|c|c|c|c|c|}
\hline \multirow[b]{3}{*}{ Parameters } & \multicolumn{12}{|c|}{ HPV-16 } \\
\hline & \multicolumn{4}{|c|}{ NLFP with $p \sim \operatorname{dunif}(-1.6,-1.2)$} & \multicolumn{4}{|c|}{ NLFP with $p \sim \operatorname{dunif}(-3,3)$} & \multicolumn{3}{|c|}{ NLFP with $p \sim \operatorname{dunif}(-5,5)$} & \multirow[b]{2}{*}{$95 \% \mathrm{CI}$} \\
\hline & mean & sd & MC error & $95 \% \mathrm{CI}$ & mean & $\mathrm{sd}$ & MC error & $95 \% \mathrm{CI}$ & mean & sd & MC error & \\
\hline$\beta_{0}$ & 2.604 & 0.026 & 0.001 & $(2.554,2.653)$ & 2.604 & 0.024 & 0.001 & $(2.557,2.650)$ & 2.604 & 0.024 & 0.001 & $(2.557,2.650)$ \\
\hline$\beta_{1}$ & 13.910 & 1.134 & 0.059 & $(11.750,16.240)$ & 13.720 & 1.045 & 0.054 & $(11.810,15.860)$ & 13.720 & 1.045 & 0.054 & $(11.810,15.860)$ \\
\hline$\sigma_{b_{0}}$ & 0.459 & 0.017 & 0.0001 & $(0.427,0.495)$ & 0.459 & 0.017 & $1.34 \mathrm{E}-4$ & $(0.427,0.495)$ & 0.459 & 0.017 & $1.34 \mathrm{E}-4$ & $(0.427,0.495)$ \\
\hline$\sigma_{b_{1}}$ & 4.738 & 0.446 & 0.020 & $(3.904,5.66)$ & 4.668 & 0.416 & 0.010 & $(2.576,3.538)$ & 4.668 & 0.416 & 0.010 & $(2.576,3.538)$ \\
\hline$\sigma_{\varepsilon}$ & 0.184 & 0.003 & $2.79 \mathrm{E}-5$ & $(0.178,0.190)$ & 0.184 & 0.003 & $2.85 \mathrm{E}-5$ & $(0.178,0.190)$ & 0.184 & 0.003 & $2.85 \mathrm{E}-5$ & $(0.178,0.190)$ \\
\hline$\rho_{b_{0} b_{1}}$ & -0.411 & 0.051 & 0.0005 & $(-0.508,-0.305)$ & -0.411 & 0.053 & $4.80 \mathrm{E}-4$ & $(-0.510,-0.304)$ & -0.411 & 0.053 & $4.8 \mathrm{E}-4$ & $(-0.510,-0.304)$ \\
\hline$p$ & -1.354 & 0.043 & 0.002 & $(-1.439,-1.266)$ & -1.229 & 0.033 & 0.001 & $(-1.293,-1.165)$ & -1.229 & 0.033 & 0.001 & $(-1.293,-1.165)$ \\
\hline \multirow[t]{3}{*}{$D I C$} & \multicolumn{4}{|c|}{-777.19} & \multicolumn{4}{|c|}{-777.4} & \multicolumn{3}{|c|}{-777.4} & \\
\hline & \multicolumn{12}{|c|}{ HPV-18 } \\
\hline & \multicolumn{4}{|c|}{ NLFP with $p \sim \operatorname{dunif}(-1.6,-1.2)$} & \multicolumn{4}{|c|}{ NLFP with $p \sim \operatorname{dunif}(-3,3)$} & \multicolumn{3}{|c|}{ NLFP with $p \sim \operatorname{dunif}(-5,5)$} & \\
\hline Parameters & mean & sd & MC error & $95 \% \mathrm{CI}$ & mean & sd & MC error & $95 \%$ CI & mean & sd & MC error & $95 \% \mathrm{CI}$ \\
\hline$\beta_{0}$ & 2.466 & 0.026 & 0.001 & $(2.415,2.517)$ & 2.466 & 0.024 & 0.001 & $(2.419,2.512)$ & 2.466 & 0.024 & 0.001 & $(2.419,2.512)$ \\
\hline$\beta_{1}$ & 11.84 & 0.639 & 0.032 & $(10.88,13.26)$ & 11.63 & 0.755 & 0.038 & $(10.22,13.28)$ & 11.63 & 0.755 & 0.038 & $(10.22,13.28)$ \\
\hline$\sigma_{b_{0}}$ & 0.4603 & 0.017 & 0.0001 & $(0.428,0.495)$ & 0.4604 & 0.017 & 0.0001 & $(0.428,0.495)$ & 0.4604 & 0.017 & 0.0001 & $(0.428,0.495)$ \\
\hline$\sigma_{b_{1}}$ & 3.227 & 0.242 & 0.008 & $(2.8,3.748)$ & 3.175 & 0.261 & 0.010 & $(2.69,3.73)$ & 3.175 & 0.261 & 0.010 & $(2.69,3.73)$ \\
\hline$\sigma_{\varepsilon}$ & 0.169 & 0.003 & $2.343 \mathrm{E}-5$ & $(0.164,0.175)$ & 0.169 & 0.003 & $2.42 \mathrm{E}-5$ & $(0.164,0.175)$ & 0.169 & 0.003 & $2.42 \mathrm{E}-5$ & $(0.164,0.175)$ \\
\hline$\rho_{b_{0} b_{1}}$ & -0.596 & 0.043 & 0.0004 & $(-0.676,-0.506)$ & -0.597 & 0.043 & 0.0004 & $(-0.677,-0.507)$ & -0.597 & 0.043 & 0.0004 & $(-0.677,-0.507)$ \\
\hline$p$ & -1.243 & 0.028 & 0.001 & $(-1.304,-1.202)$ & -1.232 & 0.034 & 0.002 & $(-1.302,-1.165)$ & -1.232 & 0.034 & 0.002 & $(-1.302,-1.165)$ \\
\hline$D I C$ & & -1232.06 & & & & -1232.770 & & & & -1232.770 & & \\
\hline
\end{tabular}

\section{Concluding Remarks}

In this paper, we proposed an extension of the fractional polynomial model discussed by Aregay et al. (2013) to non-linear fractional polynomial using a hierarchical Bayesian model. We have shown that the model can be used to calculate a subject-specific probability of being above a threshold and to predict the longterm persistence of vaccine induced anti-HPV-16/18 antibodies. The Bayesian perspective of the fractional polynomial was implemented by assuming a uniform prior distribution for the power. The NLFP is more flexible than the fractional polynomial, which assumes pre-specified fractional powers. It can easily be extended to include multiple covariates. We have conducted sensitivity analysis, establishing that the results do not depend on the prior distribution of the power. 
HPV-16

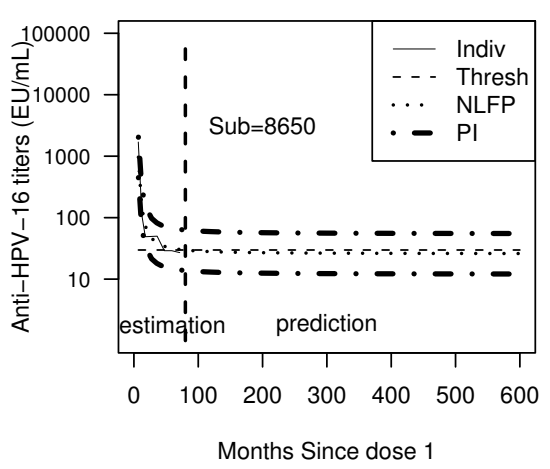

HPV-16

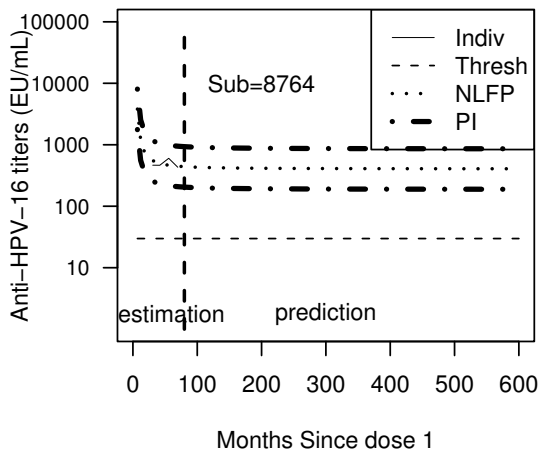

HPV-16

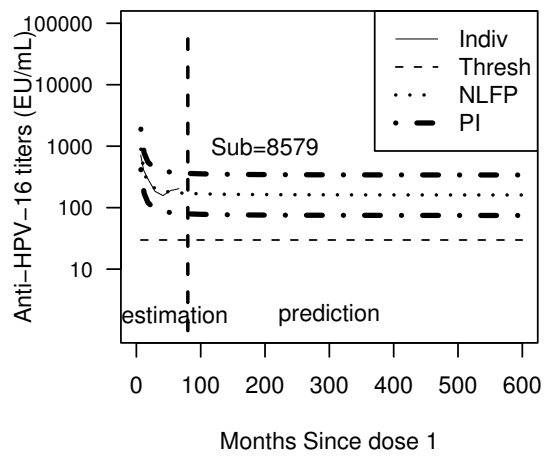

HPV-16

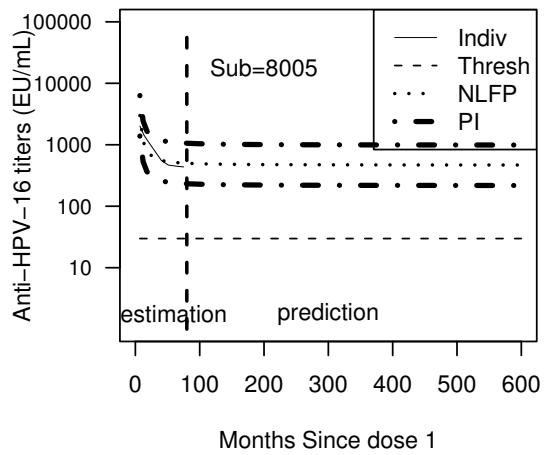

Figure 7: Long-term (50 years) prediction with posterior predictive interval of some selected subjects for anti-HPV-16 antibodies.

Furthermore, using the current method, the uncertainty of lying above a threshold can be calculated for subjects who were classified as above/below a threshold in David et al. (2009) and Aregay et al. (2013). Subjects who were classified previously as above a threshold have some uncertainty of being above the threshold also in this study (Figure 9; Figure 10).

For both HPV-16 and HPV-18, the main findings show that the posterior probability of being above the threshold value is equal to one for $97.5 \%$ subjects (380 out of 390 subjects) over 50 years. We note that one can also estimate the probability of being above a given threshold, proposed by David et al. (2009) and Aregay et al. (2013), by simulating from a normal distribution with mean $\widehat{f}\left(t_{i j}\right)$ and residual variance, $\widehat{\sigma_{\varepsilon}^{2}}$.

One of our objectives was to obtain the long-term individual prediction of being above a threshold. Hence, 
Years $=10$

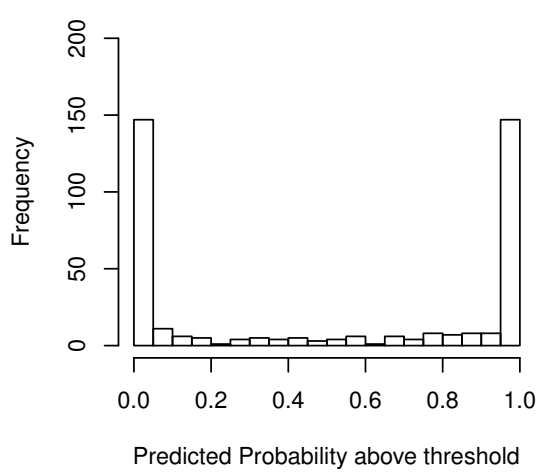

Years $=\mathbf{3 0}$

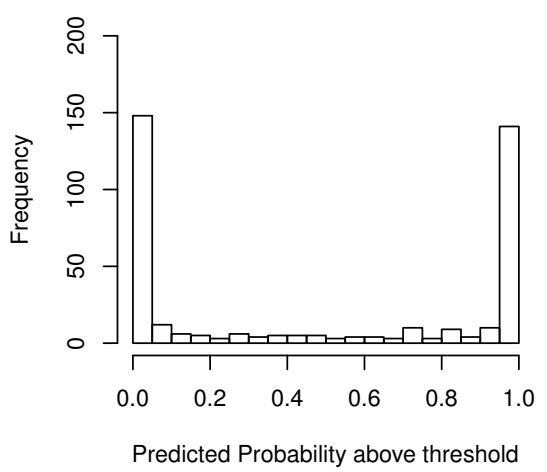

Years $=\mathbf{2 0}$

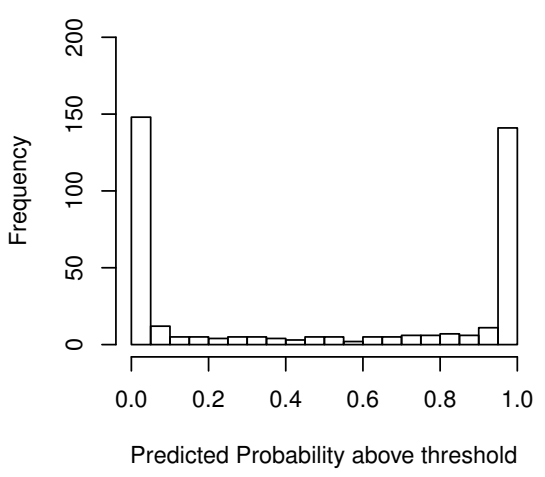

Years $=50$

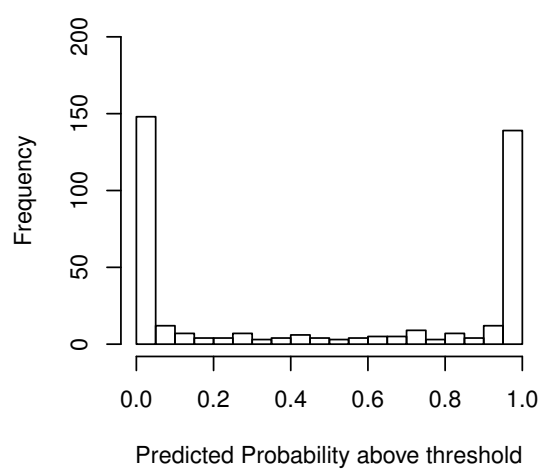

Figure 8: The posterior probability of being above threshold value 2.621, at 10, 20, 30, and 50 years for anti-HPV-16 antibodies.

the posterior individual predictive mean was calculated. It was found that 389 out of 390 subjects had posterior predicted mean above a threshold level for 50 years for anti-HPV-16 antibodies while 388 out of 390 subjects for anti-HPV-18 antibodies. If we use $\tau=2.621$, the proportion of subjects above this threshold for 50 years was approximately $48.9 \%$ for anti-HPV-16 antibodies, whereas $52.6 \%$ for anti-HPV-18 antibodies with $\tau=2.446$. These results were similar to previous findings that were obtained from the same data set by Aregay et al. (2013). We were able to show that the posterior predicted mean was above the threshold level for 50 years.

Model comparison between the non-linear fractional polynomial and power-law model was done using the Deviance Information Criterion. For both anti-HPV-16 and anti-HPV-18 antibodies, the NLFP was to be preferred. To evaluate the performance of the prediction over the estimation period, the model-based 

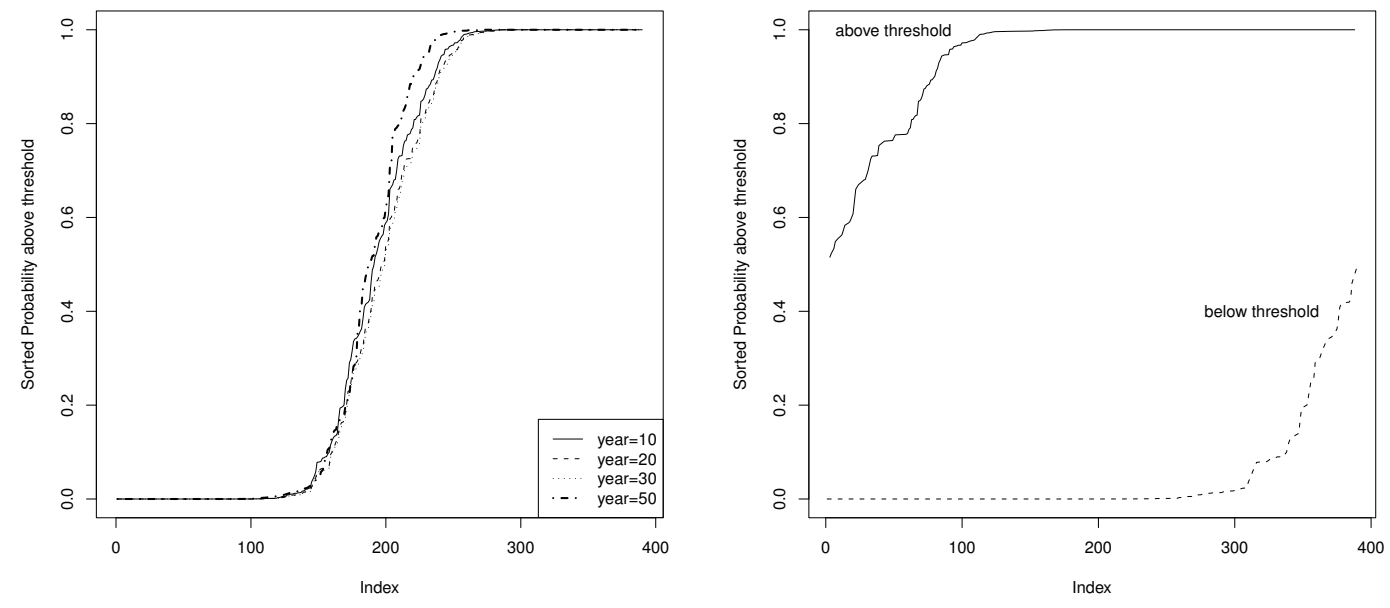

Figure 9: HPV-16: Subject-specific sorted posterior probability of being above threshold 2.621 (left panel) and posterior probability of being above threshold 2.621 over 10 years for subjects who were classified as above threshold and below threshold (right panel). The index represents the number of subjects.

proportions and observed proportions for both models were obtained. The NLFP returned proportions more similar to the observed proportion than the power-law model. Hence, in this work, the NLFP model fits to the data better than the power-law model within the follow-up period. Moreover, to assess the robustness of the inferences, the modified power-law (MPL) model was implemented. The NLFP fits the data better than the MPL model. On the other hand, the results obtained from the MPL and NLFP models are comparable (see Supplementary Web Appendix). However, this does not automatically mean it does the same outside the range of the observed data. Rather, this can be ascertained only with long-term follow up.

In summary, we have shown that the fractional polynomial framework can be extended to a non-linear fractional polynomial approach by assuming a prior distribution on the power using Bayesian approach. In this study, more than $99 \%$ of the subjects who were vaccinated with HPV-16/18 AS04-adjuvanted vaccine, had higher chance of having antibody level above the threshold level for 50 years. Moreover, we have discussed that subjects who were classified as above a threshold in David et al. (2009) and Aregay et al. (2013) approach, will not automatically be classified above a threshold in this study. We have also shown that the performance of different vaccines can be compared using the posterior probability of being above a threshold for a given time point. We would like to recommend that further work be done to define a 


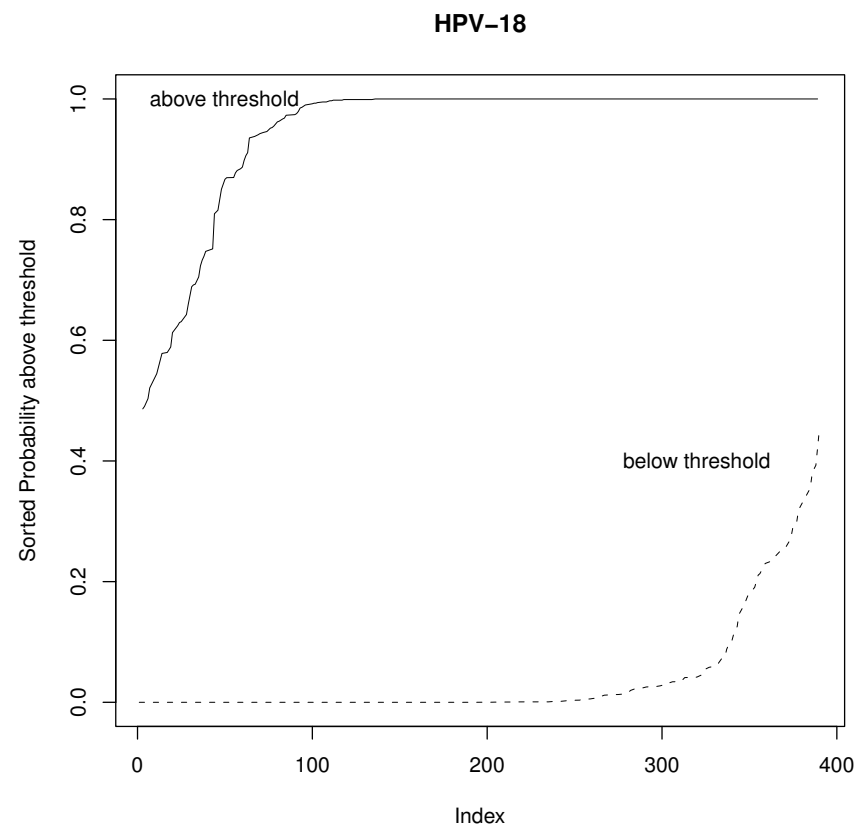

Figure 10: HPV-18: Subject-specific posterior probability of being above threshold 2.446 over 10 years for subjects who were classified as above threshold and below threshold. The index represents the number of subjects.

threshold antibody level associated with protection.

\section{Acknowledgments}

Mehreteab Aregay, Ziv Shkedy and Geert Molenberghs gratefully acknowledge support from IAP research Network P7/06 of the Belgian Government (Belgian Science Policy). The authors thank the study participants and clinical investigators from the Phase IIb primary efficacy study (NCT00689741). Finally, they thank the laboratory personnel for their contribution in performing the assays.

\section{References}

Aregay, M., Shkedy, Z., Molenberghs, G., David, M., and Tibaldi, F. (2013). Model based estimates of long-term persistence of induced HPV antibodies: A flexible subject-specific approach. Journal of Biopharmaceutical Statistics, 23, 000-000. 


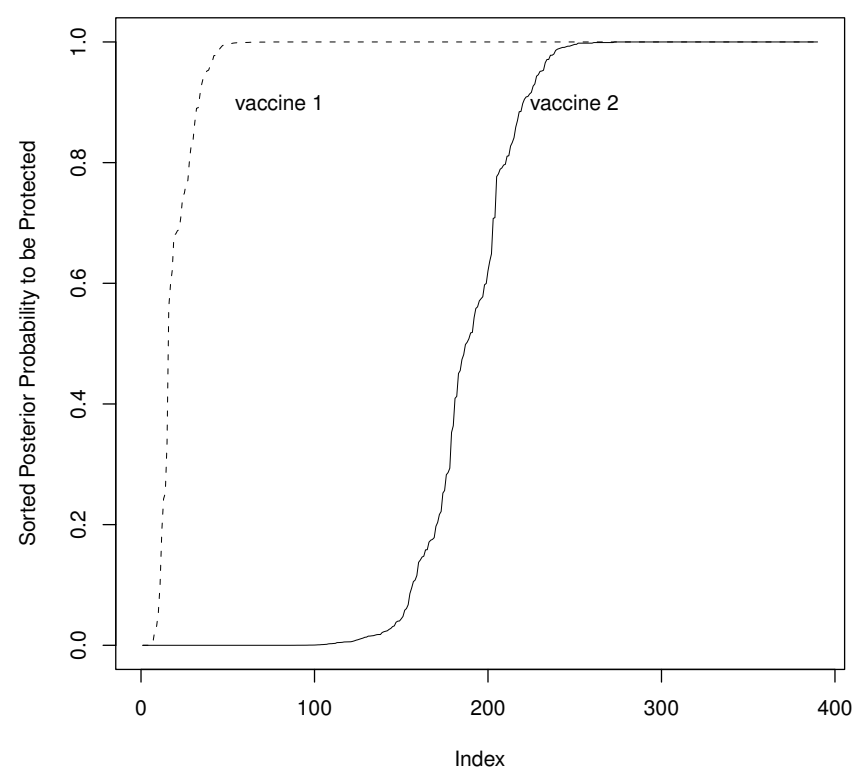

Figure 11: An illustrative probability of being above a threshold plot, which shows the comparison of two vaccines.

Auranen, K., Eichner, M., Käyhty, H., Takala, A.K., and Arjas, E. (1999). A hierarchical Bayesian model to predict the duration of immunity against Hib. Biometrics, 55, 1306-1313.

Bosch, F.X., Lorincz, A., Munoz, N., Meijer, C.J., and Shah, K.V. (2002). The causal relation between human papillomavirus and cervical cancer. Journal of Clinical Pathology, 55, 244-265.

Bové, D.S. and Held, L. (2010). Bayesian fractional polynomials. Journal of Clinical Pathology, Statistics and Computing. Epub ahead of print.

Box, G.E.P. and Tidwell, P.W. (1962). Transformation of the independent variables. Technometrics, 4, $531-550$.

Chaturvedi, A. and Maura, L.G. (2010). Human Papillomavirus and Head and Neck Cancer. In: Andrew, F.O. Epidemiology, Pathogenesis, and Prevention of Head and Neck Cancer. New York: Springer.

David, M., Van Herck, K., Hardt, K., Tibaldi, F., Dubin, G., Descamps, D., and Van Damme, P. (2009). Long-term persistence of anti-HPV-16 and -18 antibodies induced by vaccination with the AS04- 
adjuvant cervical cancer vaccine: Modeling of sustained antibody responses. Gynecologic Oncology, 115, S1-S6.

Fraser, C., Tomassini, J.E., Xi, L., Golm, G., Watson, M., and Giuliano, A.R. (2007). Modeling the longterm antibody response of a human papillomavirus (HPV) virus-like particle (VLP) type 16 prophylactic vaccine. Vaccine, 25, 4324-4333.

Gelman, A. (2006). Prior distribution for variance parameters in hierarchical models. Bayesian Analysis, 3, 515-533

Gelman, A., Carlin, J.B., Stern, H.S., and Rubin, D.B. (2004). Bayesian Data Analysis. New York: Chapman and Hall/CRC.

Gelman, A. and Rubin, D.B. (1992). Inference from iterative simulation using multiple sequences (with discussion). Statistical Science, 7, 457-511.

Goldstein, M.A., Goodman, A., del Carmen, M.G., and Wilbur, D.C. (2009). Case records of the Massachusetts General Hospital. Case 10-2009. A 23-year-old woman with an abnormal Papanicolaou smear. New England Journal of Medicine, 360, 1337-1344.

Govindarajulu, U.S., Malloy, E.J., Ganguli, B., Spiegelman, D., and Eisen, E.A. (2009). The comparison of alternative smoothing methods for fitting non-linear exposure-response relationships with Cox models in a simulation study. International Journal of Biostatistics, 5, 1-19.

Ho, G.Y., Bierman, R., Beardsley, L., Chang, C.J., and Burk, R.D. (1998). Natural history of cervicovaginal papillomavirus infection in young women. New England Journal of Medicine, 338, 423-428.

Kahn, J.A. (2009). HPV vaccination for the prevention of cervical intraepithelial neoplasia. New England Journal of Medicine, 361, 271-278.

Kirnbauer, R., Booy, F., Cheng, N., Lowy, D.R., and Schiller, J.T. (1992). Papillomavirus L1 major capsid protein self-assembles into virus-like particles that are highly immunogenic. Proceedings of the National Academy of Sciences, 89, 12180-12184. 
Long, J. and Ryoo, J. (2010). Using fractional polynomials to model non-linear trends in longitudinal data. British Journal of Mathematical and Statistical Psychology, 63, 177-203.

Muñoz, N., Boschm F.X., de Sanjose, S., Herrero, R., Castellsague, X., and Shah, K.V. (2003). Epidemiologic classification of human papillomavirus types associated with cervical cancer. New England Journal of Medicine, 348, 518-527.

Royston, P. and Altman, D.G. (1994). Regression using fractional polynomials of continuous covariates: parsimonious parametric modeling, Applied Statistics, 43, 429-467.

Royston, P. and Sauerbrei, W. (2008). Multivariate Model Building; A pragmatic Approach to Regression Analysis Based on Fractional Polynomials for Modeling Continuous Variables. New York: John Wiley \& Sons.

Shkedy, Z., Aerts, M., Molenberghs, G., Beutels, P., and van Damme, P. (2006). Modelling force of infection from prevalence data using fractional polynomials. Statistics in Medicine, 25, 1577-1591.

Spiegelhalter, D.J., Best, N.G., Carlin, B.P., and Van Der Linde, A. (2002). Bayesian measures of model complexity and fit (with discussion). Journal of Royal Statistical Society, Series B, 64, 583-616.

Sturtz, S., Ligges, U., and Gelman, A. (2005). R2winbugs: A package for running WinBUGS from R. Journal of Statistical Software, 12, 1-16.

Van Damme, P., Thoelen, S., Cramm, M., de Groote, K., Safary, A. and Meheus, A. (1994), Inactivated hepatitis A vaccine: Reactogenicity, immunogenicity, and long-term antibody persistence. $J . M e d$. Virol., 44: 446?451.

Wiens, B.L., Bohidar, N.R., Pigeon, J.G., Egan, J., Hurni, W. Brown, L., Kuter, B.J., and Nalin, D.R. (1996) Duration of Protection From Clinical Hepatitis A Disease After Vaccination With VAQTAS, Journal of Medical Virology 49:235-241.

Zhou, J., Sun, X.Y., Stenzel, D.J., and Frazer, I.H. (1991). Expression of vaccinia recombinant HPV 16 L1 and L2 ORF proteins in epithelial cells is sufficient for assembly of HPV virion-like particles. Virology, 185, 251-257. 